\title{
"Omics" and Biomedical Informatics: The Future of Pathology and Pathology Informatics. But, are we Ready for the Future?
}

\author{
Ming Chao Wong • Ray Udayan - Kwang Chien Yee
}

Published online: 23 July 2011

(C) Association of Clinical Biochemists of India 2011

\section{Introduction}

Healthcare industry is undergoing one of the most massive transformations ever seen in any other industries. There will be an escalation of challenges facing the healthcare industry in the next few decades. Among these challenges are proliferation of new technology and information management within the healthcare system. Pathology services pride itself in driving the medical changes through adaptation of new technology into the healthcare system, especially in combination with the sophisticated pathology informatics systems. The pathology informatics systems are arguably the most advanced and sophisticated information systems within the healthcare sector for many years. Some of the most exciting news in medicine such as the human genome project and the messaging and taxonomy standardisation all involve pathology services and pathology informatics [1].

In the next few decades, medical care will experience unprecedented velocity of technology advancement. Pathology services and pathology informatics will continue

M. C. Wong - K. C. Yee

School of Information Systems, University of Tasmania,

Hobart, Australia

M. C. Wong

Smart Internet Technology Cooperative Research Centre,

Melbourne, Australia

R. Udayan $(\bowtie)$

Royal Hobart Hospital, GPO Box-1061,

Hobart, TAS 7001, Australia

e-mail: udayan.ray@dhhs.tas.gov.au

to lead the transformation of medical care through genomic, proteinomics, tandem mass spectrometry and microarray technologies [1]. From the view of pathology service and pathology informatics, the next few decades are going to be exciting yet challenging. The "omics" technology and biomedical informatics will gradually merge together and be an integral part in the deep sea of laboratory medicine. These new advances will not only have significant impact in the delivery of the diagnostic and the therapeutic manoeuvres, but also in the workflow of medical practice and ethos of patient care delivery. While the technical aspect of laboratory medicine will continue to revolutionise healthcare system, the associated changes within the sociocultural context of medical practice is unclear. This study explores the issues of socio-technical interaction with advances in pathology services and pathology informatics. This study aims to provide a conceptual framework for future discussion about socio-cultural integration of technology and pathology informatics into the healthcare system to provide seamless patient-centred care delivery which would provide provider-client understanding of the service delivery system in an user friendly way.

Firstly, the study describes a hypothetical case, the technical aspect of which is achievable in the next decades by advances in biomedical informatics. By using the hypothetical case, the paper explores the socio-cultural challenges imposed by advances in technology on pathologists, clinicians and consumers. The paper reveals the barriers and challenges faced by future pathology informatics within the constrains of current organisational and professional structures of the healthcare system. It then explores the ethical, social and cultural aspects of consumers of the future. While the "omics" future might be real from technological perspective, we, the healthcare workers and consumers might not be ready then for it! 
The Year 2017: Hypotheticalfuture Healthcare System

The Patient: Mrs. Future

"My name is Mrs. Future, I walked into the hospital with chest pain, after talking to a voice recognition and recording device about my symptoms and answered a few automatically generated questions, with the irritating monotonous machine generated voice, I was taken into a cubicle. The doctor walked in and explained to me that there were a few tests called "protein something and micro something" needs to be performed. He ordered the tests near the bedside through a hand-held computer. Bloods were taken from my arm. A few hours later, I was given some paracetamol and sent home, as I was supposed to have musculoskeletal pain with 90 over percentage certainty. When I arrived home, there was an email, with all my test results with multiple numbers and bar charts as well as a bill with three zero's to follow."

\section{The Clinician: Dr. Simple}

"My name is Dr. Simple. I saw Mrs Future, who had a combination of symptoms, suggested by the clinical informatics systems with a pre-test probability of $87.346 \%$ chance of being a musculoskeletal pain and $11.243 \%$ chance of a cardiac cause. I sent off proteinomic and micro-array analysis to investigate the cardiac and musculoskeletal causes of chest pain. $2 \mathrm{~h}$ later, my hand-held computer alerted me to the test results, with a post-test probability of $96.453 \%$ chance being of musculoskeletal cause. She was therefore sent home. I don't have much idea about how the test was done and how they work out the probability, therefore, it was difficult for me to explain to Mrs. Future about any of these."

\section{The Pathologist}

"My name is Dr. Know-All. We receive the blood sample of Mrs. Future with a clinical question of differentiating between cardiac and musculoskeletal chest pain, with pre-test probability estimated by the clinical informatics systems. We ran her blood sample through state-of-the-art multi-purpose analyser for all the proteins composition of her plasma as well as the genetic composition of her cells. We then utilise a biomedical informatics system to combine the clinical and laboratory data. This data was then directly linked to a statistical equation, which derives the probability of clinical diagnosis based on her genetic probability risk of cardiac disease as well as her production of stress-related muscle specific protein. The real-time result was automatically transmitted back to the clinician, the GP and the patient at home, as well as the pharmacy decision support systems."

Is the Future Ready for us? The Omics-Laboratory

The advances in our understanding of genomic medicine, the availability of micro-array and proteinomics techniques, as well as the rapid advances in biomedical informatics and computational analysis mean that the technical aspect of the above scenario is not far away. While there are certainly issues with laboratory-clinical interface and the integration of clinical informatics systems with pathology informatics, these issues are currently being addressed through international collaborative efforts. The development of messaging and taxonomy standards, such as the development of the reference information manual (RIM) of version 3 of health level-7 classification will provide significant ground work in achieving interoperability. Technology to combine clinical and laboratory data is currently being investigated and so is the mobile wireless technology to transmit pathology data. These efforts by various organisations provide the foundation for the technological aspect of hypothetical future to become a reality.

From a laboratory standpoint, the future of pathology lies in the effective integration of informatics management and automation into its system. The shortages of skill in the workforce will worsen over the next few years. Therefore, the increasing demand of pathology services by clinicians and patients needs to be managed through the implementation of the advanced technology and increase in automation. These demographic changes in the pathology service will accelerate the implementation of technology and technology advancement. Therefore, technology of the future is almost ready for us due to various internal and external factors that accelerate its development and implementation.

\section{Are we Ready for the Future?}

The healthcare system is arguably the most complicated systems created by mankind. The difficulties with technology implementation in healthcare are usually not due to bad programming or poor implementation, but because of the need for the better technology and faster computers to fix the problem. The clinical workplace is an environment filled with complex-dynamic interaction of organisation, technology and people. The social factors and technical 
factors are co-dependent to ensure successful technology adaptation, hence the term socio-technical systems.

While the technology aspects of "omics" laboratory is advancing rapidly towards the "idealistic" hypothetical case, the socio-cultural aspects of the "omics" laboratory are less well researched. We therefore construct a conceptual framework of the multi-faceted socio-cultural aspects of "omics" laboratory through the lenses of pathologist, clinician and consumer.

\section{The Pathologists}

The "omics" era is an exciting yet challenging future for the pathologists. The technology advances in pathology laboratory and pathology informatics will ensure pathology services continue to take a leadership role in the transformation of the healthcare systems. The increase in demand and workload for pathology services, however, will create a significant challenge for pathologists. These challenges include informatics management, organisational management, workforce shortages and the burden of increasing clinical responsibility.

The "omics" future will require pathologists to have good grasp in the informatics management. The utilisation of proteinomics and genomic investigations will require better computational tools to analysis the results. Therefore, information management will become an integral part of pathology training in order to achieve biomedical and clinical informatics integration.

Secondly, the advancement in technology will exacerbate the skill shortage in pathology services. While automation will become more common in the future, the provision of the clinical decision support as well as interaction with patients in the future will mean that increasing number of pathologists and laboratory scientists will be needed.

Thirdly, the "omics" era of pathology services will inevitably lead to consolidation of the sector and centralisation of testing due to the cost of testing. Pathologists will need to work within the constrain of availability of infrastructure, yet maintaining a broad base of knowledge. Some of the socio-cultural consequences of these changes will mean that the interaction among pathology scientists and staffs might change. The hurdle the pathology practitioners would have to cross would be to ensure proper grasping of the new technology by the laboratory scientists.

\section{The Clinician}

From a clinician point of view, the omics future is daunting. There are issues with clinical autonomy, clinical responsibility, professional development, knowledge management, organisation structural changes and workflow changes as well as coping with the expectation of patients to their satisfaction.

Firstly, the clinical autonomy and professional skills have been the guarding principles of patient care for centuries. Clinicians adapt "hypothetical-deductive" reasoning, using laboratory results through Bayesian logic, in combination with anecdotal experience and evidence based decision support systems to derive diagnostic and therapeutic decisions. Within this framework, clinicians enjoy great clinical autonomy, based on their professional skills. The development of the "omics" laboratory, in combination with advanced clinical informatics, biomedical informatics systems and clinical decision support systems is a direct challenge to the clinical autonomy enjoyed by clinicians. Clinicians will need to change themselves significantly, not only to face the increased workflow, but also to equip with the understanding of the newer discovery of the underlying principles of diagnosis and management. The engagement of clinicians in the development and implementation of these technologies is essential to improve translation of laboratory medicine to bedside [1]. Clinicians need to feel safe and secure with the introduction of new technology, in order to best utilise the technology, as proposed by Maslow's hierarchy of need for organisational management and motivation.

Secondly, knowledge management will be a major issue for the clinician. The proliferation of research, technology and intervention will inevitably force the centralisation of laboratory testing to ensure cost-effectiveness. Therefore, in addition to a whole list of locally driven evidence-based protocol and guidelines, clinicians will need to have tacit knowledge about laboratory testing availability. Medical knowledge is proliferating rapidly with increasing number of research and publications. Professional development is already occupying a significant amount of clinicians' time. The development of new "omics" knowledge will necessitate clinicians to gain knowledge at an increasing speed. The translation of the knowledge to bedside will need to be of shorter interval. The new technology will need to take into account the training requirement of clinicians as well as the effective delivery of these training requirements.

Thirdly, clinicians are already struggling to remember and understand laboratory results without the "omics" complexity. Laboratory results are represented by an absolute value and a reference range. Problems arise when different reference ranges are used by different laboratory. The future pathology testing will involve testing a whole series of genes or protein expressions. The absolute values of each protein are not as important as the pattern of expressions. The advances in "omics" medicine therefore need to take into account data and knowledge representation in a way that clinicians can utilise the data quickly within the busy clinical setting. 
Fourthly, the "omics" era will indicate a change of clinical responsibility. Clinicians will share the clinical diagnostic responsibility with pathologists. Pathologists, armed with expert knowledge in "omics" will have to share the responsibility to decide which tests to perform in order to come to a diagnosis. This will shift the pendulum of clinical arena-laboratory collaboration, with pathologists playing a more central role in clinical care. The sociocultural implication of these changes will need to be managed professionally for best patient care. This would threaten the professional boundary between laboratory and clinical medicine and will have significant socio-cultural impact in healthcare.

Finally, the shifting and sharing of clinical responsibility would bring in a drastic change in managing patients' expectations. The current healthcare structure dictates the responsibility to clinicians. With the increasing role of pathologists in assisting diagnostic testing of patients, patient expectation management would become a shared role. Patient expectation brings out medico-legal responsibility. With the blurring of professional boundaries, the medico-legal framework would the need to be challenged and reformed to reflect the changes in clinical-pathological interaction.

\section{The Consumers}

From a consumer's perspective, the advances in medicine will bring about faster diagnosis and better treatment. There are, however, many issues that need to be considered for the introduction of "omics" future and seamless pathology informatics systems.

The first concern from consumer's point of view is information security, privacy and confidentiality. Consumers expect most cares to be carried out in the community setting by primary care providers. The expectation is of a seamless transmission of information to and from primary to tertiary care centre. The concern is about the confidentiality the privacy and the security. The widespread availability of digital media provides information around the world on data security and privacy. The story of hacking and identity theft is so common that hardly any consumer would have never heard of these issues. This is especially important in the "omics" era. While direct consumer data presentation might be important at one end, at another ensuring the information reaching the "right person" is difficult, especially with mobile and wireless technology.

Secondly, most consumers want to have a basic understanding of the world, especially of medical world relating to their own health. The development of digital media, internet and wireless technology has already created an environment of which consumers have no real understanding or control of the technology utilised in these devices. With the "omics" development, a medical world completely foreign and incomprehensible to most consumers will be in direct conflict to patient empowerment and patient centred care principles.

Thirdly, consumers with variability in their expectation of medical care form a diverse group. Some would want to have full access to all medical results while others trust their clinicians in doing the appropriate management. The availability of information will need to be an option accessible to the consumers. Most consumers would want to know the meaning of the pathology tests. Consumers want healthcare professionals to explain the results in an understandable way. While real-time release of data to everyone, including consumers is achievable through welldesigned technology, the outcome might be one of increase in consumer anxiety and healthcare utilisation.

Fourthly, technology often brings about unexpected consequences. The same applies to about genomic and proteinomics analysis. Examination of the genetic sequence carries a significant implication bioethically, socially and culturally. From a bioethical stand point, patient will loose individual power to decide whether they want to know the genomic structure of themselves. While from a utilitarian point of view, these analysis of human genome, through laboratory testing having therefore the potential to create data-warehouse and data-mining to discover new knowledge, is justifiable, individual right of consumers is breached. From socio-cultural stand point, the availability of genomic analysis might reveal some important yet unacceptable "facts". Do you really want to know that people you called mum and dad are not your parents after all, only because you broke your ankle and requiring some analgesia?

Finally, a trusting relationship with clinician is a healing relationship! Consumers need more than sophisticated machines and statistical probability to heal them. The "omics" laboratory will challenge the fundamental patientdoctor relationships. Consumers will increasingly use internet and other resources to search for available tests for their "suspected" conditions. The management of this relationship will be challenging for both the clinicians and the consumers.

\section{How can we Prepare Ourselves?}

There are many socio-cultural issues confronting the pathologists, clinicians and consumers with the application of "omics" technology. While the future is unpredictable and more issues will almost certainly surface during the next decades, this study develops a conceptual framework as a foundation for future discussion about the 
socio-technical impact of this technology, from the perspectives of pathologists, clinicians and consumers [1].

We can better prepare for the future if we start to understand the potential impact of this technology through the holistic socio-technical lens. There are some potential strategies to minimise the socio-cultural impacts. The first is the education and training of medical and health informatics for pathologists and clinicians. Health informatics should be included in the traditional medical courses and pathology informatics should form a part of the pathology training program. Some of the education and training programs reported great successes in health informatics and pathology informatics.

Secondly, technology implementation in healthcare should have patient-centred and socio-technical evaluation criteria. The insights into responses of consumers regarding information technology, especially implementation of informatics systems in the pathology are invaluable for the future. Furthermore, socio-technical evaluation should be a standard evaluation criteria of all technology implementation projects in healthcare. This will allow us to have a better understanding of the potential impact of technology on socio-cultural factors as well as the impact of sociocultural factors on technology implementation.

Finally, the design and the implementation phases of technology and pathology informatics systems demand early involvement of the end-users. Given the uncertainty about the impact of technology on the healthcare system, journey towards the technology implementation would be perceived as a process of learning with pathologists, clinicians and consumers. The knowledge and lesson learnt through the implementation process should be well distributed, shared among and presented widely from the perspective of pathologists, clinicians and consumers.
The socio-technical aspects of "omics" medicine presented in this study provides the foundation for future discussion. The first world pathology informatics conference provides the essential platform to engage pathologists, clinicians, consumers and pathology informatics enthusiasts into a debate as well as sharing knowledge and lessons to equip us for the challenging future.

\section{Conclusion}

The healthcare system is facing the biggest challenge and going through a rapid phase of transformation so far. The "omics" technology and pathology informatics system will lead the medical world during this transformation. There are, however, many socio-cultural issues involving pathologists, clinicians and consumers need to be addressed. This paper provides a conceptual framework which would discuss and address these socio-cultural-technical issues. Many strategies might help us to have a better understanding and prepare ourselves for socio-technical integration. Most important of all, research and discussion surrounding the socio-technical aspect of "omics" phenomena are urgently needed. The first world pathology informatics conference provides a platform where we can engage all parties to a healthy debate and discussion.

\section{References}

1. Wears RL, Berg M. Computer technology and clinical work: still waiting for godot. JAMA. 2005;293:1261-3. 